\title{
TRANSFORMANDO LA "GRAMÁTICA ESCOLAR" PARA SER MÁS INCLUSIVOS: LA EXPERIENCIA DE TRES CENTROS EDUCATIVOS
}

\author{
Cecilia Simón Rueda \\ Marta Sandoval Mena \\ Gerardo Echeita Sarrionandia \\ Universidad Autónoma de Madrid \\ Cristóbal Calero Gil Colegio Cardenal \\ Spínola (Madrid) Beatriz Núñez Gutiérrez \\ de San Miguel \\ Pilar de Sotto Esteban \\ Centro de Formación Padre Piquer (Madrid) \\ M. Montserrat Pérez García \\ Ana Belén García de la Torre \\ Centro educativo Ponce de León (Madrid)
}

\begin{abstract}
RESUMEN: El objetivo de este artículo es compartir con el lector algunas de las primeras reflexiones realizadas por los investigadores y profesorado de tres centros educativos españoles que participan en el proyecto europeo Teaching diverse learners in (School)Subjects), coordinado por Kerstin MerzAtalik y Heike Tiemann (University of Education Ludwigsburg). Desde nuestro planteamiento, siguiendo algunas de líneas centrales de acción propuestas en este momento, si realmente queremos avanzar hacia un sistema educativo y unas prácticas escolares con capacidad para acoger, reconocer y responder con equidad a toda la diversidad del alumnado, sin restricciones ni exclusiones en cuanto a ese "todos", entonces no queda más remedio que cambiar radicalmente la "gramática escolar" existente. Nuestra finalidad será mostrar algunos de los elementos centrales que se han cambiado en los tres centros españoles participantes, para tratar de asegurar el equilibrio entre calidad y equidad educativa desde la realidad de cada uno de ellos. A partir del análisis de las principales barreras y desafíos para avanzar hacia una educación cada vez más inclusiva, se delimitan algunos de los elementos que ha sido necesario transformar en los centros educativos para acortar la distancia entre los valores declarados y las prácticas específicas.
\end{abstract}

PALABRAS CLAVE: Educación inclusiva, prácticas docentes, gramática escolar, transformación escolar. 


\title{
TRANSFORMING THE “GRAMMAR SCHOOL" TO BE MORE INCLUSIVE: THE EXPERIENCE OF THREE SPANISH SCHOOLS
}

\begin{abstract}
The aim of this article is to share some of the early reflections made by the researchers and teachers from three Spanish educational centers that are participating in the European project Teaching diverse learners in (School) Subjects) coordinated by Kerstin Merz-Atalik and Heike Tiemann (University of Education Ludwigsburg). From our point of view, if we really want to make progress toward an educational system and schools with capacity to respond with equity and fairness to the student diversity, we will have to radically change the existing "grammar school". Our purpose will be to show some of the key elements that have been changed in the three participating centres, to try to ensure the balance between quality and educational equity from the reality of each one. Based on the analysis of the main barriers and challenges to move toward an inclusive education, here are listed some of the elements that have been necessary to transform in these educational institutions in order to shorten the distance between the declared values and specific practices.
\end{abstract}

KEYWORDS: Inclusive education, practices teaching, grammar school, improvement school.

Recibido: 18/05/2015

Aceptado: 30/05/2015

Correspondencia: Marta Sandoval Mena, Universidad Autónoma de Madrid, Facultad de Formación del Profesorado y Educación, Departamento de Psicología Evolutiva y de la Educación, C/ FranciscoTomás y Valiente 3, 28049 Madrid. Email: marta.sandoval@uam.es.

\section{INTRODUCCIÓN}

Cambiar la realidad de los centros para avanzar hacia culturas, políticas y prácticas educativas que sostengan una perspectiva más inclusiva hacia la diversidad humana, es algo que se debe construir día a día, no sin gran esfuerzo y enfrentando los dilemas que inevitablemente forman parte del proceso (Norwich, 2008). La complejidad de esta tarea se pone claramente de manifiesto cuando delimitamos hacia qué ámbitos deben dirigirse las acciones que, de una forma articulada, se tienen que poner en marcha para avanzar en esta dirección.

De acuerdo con Booth y Ainscow (2011), destacamos tres ámbitos de actuación interrelacionados. Por un lado, la inclusión como proceso de incremento de la participación de todos los miembros de la comunidad educativa y reducción de la exclusión (tanto en la escuela como en la comunidad). Por otro lado, la inclusión como proceso de transformación y construcción de espacios y sistemas (procedimientos, políticas, leyes) que promuevan esta participación y que, por tanto, incrementen su capacidad para responder a la diversidad garantizando la equidad. 
Este es, precisamente, el énfasis que la propia UNESCO (2005), hace a la hora de entender la educación inclusiva como la perspectiva que nos ayuda a reflexionar en torno a cómo transformar los sistemas educativos y otros entornos de aprendizaje con la finalidad de que estos respondan a la diversidad de los estudiantes.

Por último, la inclusión como el proceso de llevar a la acción los valores que la sostienen. Este es uno de los aspectos que fundamentan la última edición del Index for Inclusión de Booth y Ainscow (2011). Desde su planteamiento, la educación inclusiva no es una innovación educativa sin más; es una cuestión de valores, un ámbito en el que los dilemas y desacuerdos también son frecuentes y forman parte del proceso. Por ello, deben afrontarse desde un diálogo igualitario (Flecha, 2009) y entenderse como una oportunidad para pensar juntos en la escuela que queremos, en el contexto del proyecto social que anhelamos.

Los valores son la base de todas las acciones y planes de acción, de todas las prácticas en las escuelas y de todas las políticas que modelan las prácticas. Por lo tanto, se pueden considerar que todas las acciones, prácticas y políticas son la encarnación de los razonamientos morales. No podemos adoptar un comportamiento correcto en la educación sin comprender, en cierto modo, los valores de los que nacen nuestras acciones. Por lo tanto, el desarrollo de la inclusión nos implica a nosotros mismos a la hora de hacer explícitos los valores que subyacen a la inclusión de la mejor manera posible. (Booth, 2006: 212).

Por ello, hacer explícitos, compartir los valores y analizar hasta qué punto nuestras acciones son coherentes o reflejas esos valores, poniendo en marcha procesos para acortar esa distancia es, en esencia, la finalidad del Index o de cualquier otra guía en esta dirección. Éste es el marco en el que se circunscribe el trabajo que se presenta, y que forma parte de los análisis iniciales realizados en el contexto de un proyecto financiado por la Unión Europea (Lifelong Learning Programme Comenius Network, 541969-LLP1-2013-1-DE-Comenius-CNW), titulado Teaching diverse learners in (School)Subjects). Este proyecto, coordinado por Kerstin Merz-Atalik y Heike Tiemann (University of Education Ludwigsburg), cuenta con la participación de universidades y centros escolares de seis países europeos (Alemania, España, Islandia, Lituania, Luxemburgo y Suecia): University of Education Ludwigsburg (Alemania), Universidad Autónoma de Madrid (España), Geschwister-Scholl-Schule (Alemania), Landesinstitut für Schulsport, Schulkunst und Schulmusik (Alemania), University of Iceland Reykavik (Islandia); Siauliai University (Lituania), Zokniu Progymnasium Siauliai (Lituania); Sandoros Progymnasium (Lituania), University of Luxembourg (Luxemburgo), University of Boras (Suecia). Los tres centros educativos españoles que participan en el estudio son: centro de Formación Padre Piquer, colegio Cardenal Spínola y centro educativo Ponce de León.

Este proyecto pretende establecer un nexo entre teoría y práctica. Por ello, una parte importante de sus objetivos es crear una plataforma de discusión, reflexión e intercambio de experiencias entre los formadores de futuros maestros y maestras en la universidad, profesorado y equipos directivos de diferentes escuelas, representantes de la administración educativa, expertos de diferentes organismos internacionales relacionados con la promoción de la educación inclusiva, etc. Además, se pretende mostrar ex- 
periencias que permitan mostrar el vínculo entre la teoría y las prácticas desarrolladas en los centros. El foco del mismo es reflexionar y mostrar evidencias en torno a cómo garantizar una articulación justa de la presencia el aprendizaje y la participación de todo el alumnado, sin exclusiones, teniendo presente las materias específicas del currículo. Lo que tenemos muy claro es que las prácticas segregacionistas son uno de los principales factores de exclusión dentro de los sistemas educativos europeos, lo cual genera, a su vez, exclusión social y educativa (INCLUD-ED Consortium, 2011: 19).

Comprometerse con una perspectiva inclusiva implica asumir la tarea de pensar y recoger evidencias (como insisten Ainscow y West, 2008) que nos permitan responder a cuestiones tales como hasta qué punto la cultura del centro (nuestra perspectiva sobre inclusión o sobre la diversidad humana, por ejemplo), la forma de organizarnos (espacios de coordinación y reflexión conjunta por parte del profesorado, la implicación en este proceso del alumnado, las familias u otros miembros del entorno comunitario, lo que hacemos para apoyar a los que lo necesitan, etc.) o el currículo escolar (qué, cómo y cuándo enseñar y evaluar), se configuran o no, como facilitadores del aprendizaje y el rendimiento de todo el alumnado tal y como están concebidos y concretados en la actualidad. Supone plantearnos si contribuyen o, por el contrario, debilitan los esfuerzos por construir un sistema donde no se entienda equidad sin calidad.

Desde nuestro planteamiento, siguiendo algunas de líneas centrales de acción bien recogidas en la literatura internacional al respecto (Booth y Ainscow, 2011; Foreman, 2011; Powell, 2014; Slee, 2011; UNESCO, 2005; Winter y O'Raw, 2010), si realmente queremos avanzar hacia un sistema educativo y unas prácticas escolares con capacidad para acoger, reconocer y responder con equidad a toda la diversidad del alumnado, sin restricciones ni exclusiones en cuanto a ese "todos", entonces no queda más remedio que cambiar radicalmente la "gramática escolar" existente (Tyack y Cuban, 2001). En este sentido cabe señalar, por ejemplo, que "la escuela" que mayoritariamente conocemos (y por consiguiente su gramática), no ha estado pensada para "acoger, reconocer y responder con equidad a la diversidad del alumnado", sino que más bien ha sido selectiva en cuanto a quién puede (o ha podido) incorporarse a ella y, por ello, también segregadora respecto al resto de alumnado que no ha accedido a la misma, así como homogeneizadora en cuanto a la "respuesta" (formas de enseñar y evaluar) ofrecida a los que conseguían estar o permanecer en ella.

En este trabajo compartiremos precisamente con el lector algunas de las reflexiones y prácticas llevadas a cabo por las comunidades educativas de tres centros escolares españoles que participan en el mencionado proyecto y que bien cabe calificar como de "rompedoras", al menos, de algunos de los aspectos más nucleares de esa gramática al uso. Finalmente, el trabajo conjunto entre "investigadores y prácticos" nos ha permitido también reconocer y explicitar algunas lecciones aprendidas en este "viaje de transformación."

\section{PONIENDO NUESTROS VAlORES EN ACCIÓN: ¿QUÉ ESTÁ MARCANDO la DIFERENCIA EN LOS CENTROS?}

Al igual que toda lengua tiene una gramática formal que determina las normas, principios y supuestos que estructuran la comunicación con ella, cabría decir que 
"la escuela" también tiene una gramática (desarrollada y aprendida por sus "usuarios" habituales: profesorado, alumnado, familias, administración, etc.), aprendida, sobre todo, de manera implícita (también enseñada en la mayoría de los centros de formación del profesorado), que estructura lo que en ella se viene haciendo "desde siempre": qué se enseña, cómo, en qué espacios, con qué profesorado, con qué expectativas, y un largo etc., que no procede detallar porque, finalmente, tiene que ver con todo el sistema educativo.

Transformar esa realidad es muy complejo, porque lo que ocurre "dentro de las puertas de la escuela", está íntimamente interconectado con lo que ocurre "más allá de esas mismas puertas", configurando lo que Ainscow, Dyson, Goldrich y West (2012) han denominado como una compleja "ecología de la equidad". Ello debe hacer pensar a todo el mundo en la necesidad imperiosa de poner en marcha de estrategias de intervención educativas multidimensionales (no exclusivamente en el ámbito de la educación formal), para hacer frente a la inequidad En este sentido, muchos trabajos realizados nos muestran, por ejemplo, que es necesario complementar el esfuerzo que se realiza dentro de una escuela para transformarse y ofrecer una educación de calidad para todos los estudiantes, con otros esfuerzos, de carácter local y supralocal que deben ir dirigidos a establecer redes de apoyo entre centros, así como lazos con las comunidades en la que se encuentra cada centro. Evidentemente, como también hemos señalado, estos esfuerzos deberían ir de la mano de políticas nacionales encaminadas a la creación de una sociedad más justa (Simón y Echeita, 2013).

Los tres centros que se muestran y analizan, y que están insertos en un proceso de mejora e innovación sistemático, que no tienen un punto final, sino que se configura más bien como una "historia interminable", son los siguientes: Centro de Formación Padre Piquer, el centro educativo Ponce de León y el colegio Cardenal Spínola. Los tres centros son concertados y están ubicados en la Comunidad de Madrid.

El Centro de Formación Padre Piquer (a partir de ahora CFPP) tiene los niveles de Educación Secundaria Obligatoria, Bachillerato, Programas de Cualificación Profesional Inicial, Formación Profesional Básica, Formación Profesional Específica de Grado Medio y Grado Superior y Formación para el empleo. En el Primer Ciclo de la ESO un $51 \%$ de alumnos proceden de familias inmigrantes, un $37 \%$ presentan desfase curricular, un 30\% son considerados "alumnos de compensatoria" y un 9\% considerados con "necesidades educativas especiales"1. El colegio se encuentra ubicado en el barrio de La Ventilla de Madrid. El estatus socioeconómico de las familias es medio-bajo.

El Centro educativo Ponce de León (a partir de ahora CEPL) que cubre las etapas de Educación Infantil hasta Bachillerato, cuenta, además, con un centro de Educación Especial, un Centro Ocupacional y un Centro Especial de Empleo. Atiende alumnado sordo y oyente, en un porcentaje de un tercio de alumnado sordo. En lo que respecta a las etapas de educación infantil, primaria y secundaria "ordinaria" se trata de un "centro de integración preferente de sordos" y en las aulas hay una ratio de 5 alumnos sordos y 15 oyentes con comunicación por Lengua de Signos Española (LSE) y lengua castellana. El centro está ubicado en la zona sur de la Comunidad de Madrid, en el distrito de Villaverde-Usera en el barrio de Orcasitas, un barrio con

1. Aunque somos críticos con el uso de esta terminología, la utilizamos para facilitar la comunicación con el lector. 
un nivel socioeconómico medio-bajo y, en el que viven familias en situaciones de gran precariedad económica, con un alto índice de inmigración. Más del 50\% de las familias solo poseen estudios obligatorios o no tienen estudios, más del $30 \%$ están en paro, un $27 \%$ son inmigrantes y un $7 \%$ son de etnia gitana. Además, el $3 \%$ de los padres también tiene deficiencia auditiva.

El Colegio Cardenal Spínola (a partir de ahora CCS) cuenta con tres unidades desde Educación Infantil hasta $4^{\circ}$ de ESO, y dos unidades en los dos cursos de Bachillerato. Se trata de un centro en el que se encuentra escolarizado un porcentaje significativo de niños y niñas con discapacidades (7\% de alumnos con dictamen de "necesidades educativas especiales" y $7 \%$ de alumnos sin dictamen pero con dificultades de aprendizaje relevantes). Hay un porcentaje escaso de alumnado perteneciente a otros colectivos en riesgo de exclusión. Está ubicado en la zona de Chamartín donde predominan las familias de clase media, media/alta. El alumnado es, sin embargo, diverso en cuanto a recursos económicos debido a la política de apoyo en forma de becas de la Congregación de Esclavas del Divino Corazón y la Fundación Spínola.

La experiencia compartida de las tres instituciones sobre las que se basa este análisis, es que aun haciéndolo en distintos contextos socioculturales, con distintas estrategias, empezando desde distintos puntos y con distinta intensidad, todos ellos están implicados de forma activa en la tarea de ir cambiando elementos sustantivos de la gramática escolar al uso, lo que les ha llevado a pensar y hacer cosas distintas, entre los cuales, en esta primera fase del proyecto hemos destacado siete. A continuación comentaremos brevemente cada elemento y, en cada caso, haremos alusión a prácticas concretas que se desarrollan en uno o varios de estos centros:

\section{a) Equipos docentes que saben buscar y cuidar los momentos para la reflexión y el análisis de sus prácticas y de la relación de estas con los valores inclusivos que quieren mantener}

El desafío y el dilema de la diversidad, que es el núcleo de la educación inclusiva, tiene que ver con reconocer que vivimos en un mundo donde la diversidad de formas de ser, sentir, aprender, participar, amar o creer, entre otras muchas, es la norma y donde la creciente y compleja interdependencia de un mundo globalizado nos conduce a la imperiosa necesidad de aprender a valorar dicha diversidad y a convivir respetuosa, solidaria y dignamente con ella (Echeita, Simón, Urbina y López, 2013: 330).

Sin duda, la escuela, es uno de los contextos de referencia donde los estudiantes aprenden precisamente a valorar, cómo vivir en y gracias a la diversidad, y a pensar en qué tipo de mundo les gustaría vivir a este respecto. En este sentido, desde los centros se asume que la educación inclusiva es, en definitiva, una cuestión de valores y principios éticos. Son estos los que, de acuerdo con Booth y Ainscow (2011), como señalamos en la introducción, sostienen la puesta en marcha y el desarrollo de este complejo proceso de continua mejora que supone la educación inclusiva. Los procesos de reflexión y discusión de los equipos docentes de estos centros se centran, en gran medida, en la relación entre estos valores y sus distintos sistemas de prácticas. En relación con este aspecto, se procura atender a la implementación y mejora de los tiempos para la coordinación y reflexión conjunta del profesorado sobre sus prácticas 
y políticas educativas en materia de equidad/atención a la diversidad/inclusión. Así, por ejemplo, en el CEPL se han delimitado cinco espacios en los que se promueve la reflexión en el centro: a) reuniones semanales de los equipos de profesionales de cada etapa (se tratan temas organizativos y de convivencias y se Ilegan acuerdos); b) reuniones pedagógicas quincenales para tratar y profundizar en los temas, generalmente relacionados con necesidades de formación continua, expresadas por el profesorado y delimitados al comienzo del curso; c) reuniones semanales o quincenales con el Departamento de Orientación (trabajadora social, orientadora y tutoras) para tratar todos los aspectos relacionados con el alumnado; d) reuniones de incidentes (una o dos veces al trimestre) en las que participan todos los profesionales que trabajan en el aula con la finalidad de llegar a acuerdos conjuntos relativos al grupo o a alumnos o alumnas individuales, que se comparten con el resto de compañeros en una reunión de evaluación, e) reuniones semanales de seguimiento de la llamada "educación combinada" para asegurar la transición y coherencia de una etapa a otra y programar actividades y materiales de forma conjunta.

Como resultado de estos procesos de reflexión observamos cómo tienden a ser vigilantes con el uso y las implicaciones de las prácticas de categorización que afectan en particular al alumnado considerado con "necesidades educativas especiales" y que, cuando te descuidas, terminan condicionando negativamente las expectativas de su profesorado, de sus compañeros y no pocas veces las de sus propias familias. Implica atender a las necesidades de cada alumno o alumna independientemente de categorías prefijadas que tratan de agruparles. Como insisten en el CCS, ningún alumno o alumna es segregado por motivos de capacidad (ni ningún otro).

Por otro parte, los centros tratar de romper el círculo vicioso de "profecías autocumplidas" en relación con el bajo rendimiento de un alumnado que, por efectos de las expectativas negativas hacia él, ha recibido una enseñanza empobrecida, poco estimulante o conformada con "mínimos". En el CCS se evitan medidas favorecedoras de estas profecías autocumplidas tales como no promociones, materiales educativos diferentes o grupos de trabajo por niveles.

Otro aspecto que cabe destacarse, es la revisión del papel de los "servicios/departamentos de orientación", más volcados en el asesoramiento para la mejora de las prácticas educativas y una adecuada estructuración del apoyo escolar. Así, tanto en el CCS como en el CFPP, el modelo de orientación se ha transformado por completo. El rol del orientador es diferente, renuncia a su "papel de experto", para ser un compañero que apoya para asegurar el aprendizaje de todo el alumnado. Apoyan al aula, a la familia y al docente y no tanto a un grupo específicos de alumnos o alumnas por sus "necesidades especiales".

\section{b) Diferentes formatos de trabajo colaborativo y cooperativo, tanto entre el alumnado, como entre el profesorado}

Una visión compartida por los centros participantes en el proyecto es que no podemos hablar de inclusión sin superar la visión limitada y restringida de apoyo como algo restringido al trabajo de determinados profesionales y sobre un determinado grupo de alumnos "especiales". Tratan de introducir un concepto de apoyo mucho más amplio y sistémico, que se va más allá del apoyo que podemos considerar "directo" 
por parte de profesionales concretos, aunque sin duda este es importante, siempre y cuando se revise cómo se realiza desde el marco de la inclusión (ver Sandoval, Simón y Echeita, 2012).

Así, se considera que apoyo también lo pueden proporcionar otros muchos miembros de la comunidad escolar de muy diversas formas (Echeita, Simón, Sandoval y Monarca, 2013): cuando el docente planifica las actividades pensando en todo su alumnado; cuando los estudiantes se ayudan entre sí trabajando cooperativamente; cuando el profesorado se apoya mutuamente (programación de sus clases, observación mutua, en forma de equipos de veteranos que atienden las dificultades de los que tienen menos experiencia...), cuando se cuenta con la participación de las familias y otros miembros de la comunidad, etc.

En todos los centros, el agente esencial de la inclusión es el tutor de referencia, no el profesor de apoyo con diferentes ámbitos de colaboración entre profesionales, alumnado y familias.

En este marco hay que resaltar algunas prácticas singulares como la que lleva a cabo el CFPP a través de su proyecto denominado Aulas Cooperativas Multitarea (http://www.padrepiquer.es/images/videos/videoacm). En estas aulas se integran dos elementos: el aprendizaje cooperativo y la realización de diferentes tareas en el aula. Se trata de un aula flexible, en la que se respetan los distintos ritmos de aprendizaje, que permite múltiples agrupamientos, facilitando, por tanto, que tres profesores estén trabajando simultáneamente. Se trabaja mediante lo que denominan ámbitos como el ámbito SCL (sociales, lengua e inglés), el ámbito CT (ciencias, matemáticas y tecnología), y el ámbito artístico (plástica con SCL y CT) con la presencia simultánea de los profesores de dichas materias en el aula. Para ello, los horarios se han flexibilizado y organizado en torno a bloques de 2-3 horas.

También en el CCS y en el CEPL se utilizan diferentes estrategias de aprendizaje cooperativo. Por ejemplo, en el CEPL se buscan estrategias de apoyo cruzado entre alumnos, cuidando especialmente la organización de las mesas de trabajo, de forma que siguiendo las directrices de Kagan y Kagan (2009), siempre tienen próximo a un compañero al que pueden ayudar y, además, tú también puedes ser ayudado por otro, en la medida de lo posible. En el CCS están probando, parece que con muy buenos resultados, una estructura de trabajo cooperativo en tríos, que rompe con el criterio habitual de trabajar en parejas o equipos de cuatro.

Pero la utilización de estas estrategias cooperativas no solo se utiliza como herramienta de trabajo con el alumnado, sino también con otros miembros de la comunidad educativa. Así, en el CCS el claustro docente se estructura sobre las bases de grupos cooperativos y el trabajo en los claustros se hace a través de los elementos centrales de la cooperación. En este centro se trata de potenciar otros elementos de cultura cooperativa como son las tertulias dialógicas, los grupos interactivos, o los padrinos de lectura, propios del proyecto Comunidades de aprendizaje (http://www.comunidadesdeaprendizaje.net/). De la misma forma, en el CFPP se utiliza esta estrategia en las reuniones con las familias, lo que les permite mostrar cómo trabajan con sus hijos e hijas.

En el marco de esta misma "cultura colaborativa", el profesorado de apoyo participa plenamente en las aulas, muchas veces en forma de co-enseñanza con los tutores o profesores de materia. Un sentir compartido por los centros es que la responsa- 
bilidad de la educación de los estudiantes es compartida y el "tutor" lo es de todos los alumnos y alumnas. Hablamos de que el profesorado del aula no delegue la responsabilidad del trabajo con el alumnado considerado con "necesidades educativas especiales" en el profesorado calificado, a su vez, como "especialista" (profesorado de pedagogía terapéutica, audición y lenguaje, de compensatoria), a quien en otros contexto escolares se le atribuye un saber experto para el trabajo escolar con ese alumnado. En el CCS se considera que el agente esencial de la inclusión es el profesor de referencia, no el profesorado de apoyo.

Como hemos mencionado en el CEPL podemos encontrar en el aula varios profesores que trabajan como cotutores. Son dos profesores en el aula que trabajan con el mismo estatus. Lo mismo ocurre con el CFPP, al organizar las materias por ámbitos que se imparten en la misma aula y no por materias que se trabajan en aulas separadas. Además, la presencia del Departamento de Orientación dentro del aula (en la línea también de cómo se entiende el trabajo de estos profesionales en el CCS), ha sido fundamental para la inclusión de los alumnos considerados con n.e.e. en el ritmo del aula. Se intenta que estos no abandonen el aula para ser "tratados individualmente" sic, sino incluirlos en el mayor número de actividades junto con el resto de sus compañeros. La presencia de tres profesores en el aula y el profesor de apoyo facilita mucho esta estrategia. Precisamente, en el CCS, el profesorado de apoyo es un apoyo más al docente, al tutor y a la familia para lograr la inclusión. Interviene en la adaptación del aula y en el desarrollo de las clases así como en el acompañamiento familiar, pero nunca interviene de modo específico o exclusivo con un alumno o alumna. Se trata, en definitiva, de cambiar las preguntas asociadas al desafío de la diversidad. En lugar de preguntarnos "qué le pasa a este alumno o alumna, qué problemas tiene, quién puede hacerse cargo o "apoyarle", ahora las preguntas son otras: qué puede hacer este alumno hoy con ayuda, cómo puedo adaptar el aula a esa necesidad.

Cuando se ha adquirido un buen clima de confianza, el trabajo conjunto de un equipo docente en la misma aula también sirve como evaluación unos de otros, pues observándose pueden proponerse mejoras en las prácticas diarias que siempre repercuten, al final, en un mejor clima de aprendizaje en el aula.

\section{c) Formas de organización de los contenidos y abordar su aprendizaje de forma más globalizada, motivadora y con mayor capacidad de ajuste a la diversidad de capacidades, intereses o ritmos de aprendizaje del alumnado}

Hablamos de apuestas por formas de enseñar que atrapan la curiosidad y la motivación. Aulas ricas en estímulos y en oportunidades de elección, con materiales variados, con uso frecuente de las TIC y diseñadas pensando en todos los alumnos y alumnas del aula.

Por eso CEPL ponen en marcha lo que denominan Proyectos de investigación que trabajan en todas la etapa de Educación Infantil y Educación Primaria. La finalidad es aprender investigando y a partir de los intereses y deseos del alumnado. Se considera que todos los alumnos estarán preparados para hacer propuestas de trabajo, manifestar sus intereses o mostrar lo que saben, lo que se logra a través de proyectos que fomenten la curiosidad, las ganas de saber y de aprender del otro, la colaboración entre todos y la autonomía. En el proceso de elección de los proyectos participan 
todos, se realiza en la Asamblea en la que están presentes los dos cotutores; parten de sus intereses y motivación. Se elabora con ellos el Índice del proyecto (qué saben, qué quieren aprender y cómo lo van hacer (Hernández, 2002). El resultado es que los alumnos están muy motivados ya que ellos guían el proceso y porque se cuenta también con la colaboración de las familias en su desarrollo. En la ESO se desarrolla durante todo el curso el "Proyecto Créate", en el que participan de forma conjunta las áreas de Plástica, Tecnología y Matemáticas.

Otro ejemplo en esta dirección es la forma en la que el CFPP organiza las asignaturas tradicionales y que las agrupa, como hemos señalado anteriormente, en ámbitos de aprendizaje; así Lengua, Sociales e Inglés conforman el ámbito sociolingüístico y matemáticas, ciencias y tecnología el científico-matemático o tecnológico. Al ser un ámbito, es mucho más sencillo trabajar por proyectos globalizadores, con un aprendizaje más transversal. Por ejemplo, los alumnos de $2^{\circ}$ ESO realizan un recorrido histórico por el Madrid de los Austrias con la correspondiente explicación teórica del profesor durante el paseo. A su regreso y, aprovechando que en Lengua están estudiando el diálogo, tendrán que elaborar por grupos un acto de una obra teatral cuyos personajes y contenido tenga que ver con la época de los Austrias. El grupo que se anime, podrá presentarlo en inglés y en español. Finalmente, lo representarán en el Salón de Actos para sus compañeros del aula. Al ser un producto elaborado por ellos mismos y no establecido por el profesor, son los alumnos los que, conscientes de sus capacidades y limitaciones, deciden entre todos qué papel representa cada uno, etc. Partiendo de una actividad motivadora, como es el paseo histórico por Madrid, terminan elaborando un producto propio con los contenidos aprendidos.

Estos centros han prestado una atención muy especial al análisis de las metodologías docentes con la finalidad de garantizar el aprendizaje y la participación de todos los alumnos y alumnas del aula. Esto es, se evitan metodologías poco diversificadas y muy de "talla única", es decir, lo mismo para todos, en donde es el alumno el que debe "seguir el ritmo y ajustarse", de forma que los problemas o dificultades, cuando aparecen, "son del alumno", y es él el que debe cambiar. Lo que hace complejo un verdadero ajuste a las necesidades educativas de algunos alumnos o alumnas.

Así, una estrategia de gran valor para facilitar el aprendizaje y la participación de todo el alumnado es el trabajo por rincones en infantil y por zonas en primaria y la combinada (EP/EBO). El aula se organiza por zonas en cada una de las cuales los alumnos trabajan una determinada área curricular. Se trata de espacios estables que permiten una distribución espacial de aula y una mejor estructuración y aprovechamientos de los materiales. Esta organización ofrece la posibilidad de elegir, de ser autónomos, de aprender cada uno a su ritmo. Permite a su vez al docente, a través de la observación y la escucha activa, el reajuste de las actividades, la detección de dificultades o conflictos y la evaluación no solo del trabajo sino del proceso de puesta en marcha (¿saben trabajar autónomamente, organizan el material antes de trabajar...?).

Por otro lado, por ejemplo, en el CEPL, al igual que en el CFPP no hay libros de texto, son los profesores de referencia del aula y profesorado de apoyo los responsables de la creación de materiales según el proyecto elegido, pensando en cada alumno, en sus necesidades. En el CEPL lo denominan "libro importante" y en el CFPP "cuadernos de aprendizaje". 
Respecto a la utilización de las nuevas tecnologías, en el CEPL todas las aulas poseen pizarra digital y ordenadores. También se cuenta con tablet pc que les permite hacer zonas y explicaciones muy interactivas y visuales, lo cual es un aporte fundamental para todo el alumnado. Además, utilizan blog en las aulas que se actualiza constantemente y que, además, permite hacer partícipes a las familias del trabajo continuo que se realiza.

De la misma forma, en el CFPP se intenta que los alumnos puedan aprender de maneras diferentes para que, al final, Ileguen al mismo objetivo. Así, se entiende que habrá alumnos con mayor inteligencia visual-espacial a los que les ayude ver imágenes sobre el tema de estudio, para ellos las aulas cuentan con un proyector, pantalla y 16 equipos informáticos dentro de la propia clase que ellos pueden usar libremente; otros tendrán una mayor inteligencia lingüística y utilizarán los diferentes libros y recursos que hay en las bibliotecas de aula junto con sus guías de aprendizaje (en sustitución de los libros de texto). La propia fisonomía del aula es diferente a la tradicional, se han eliminado los tabiques entre clases y aunque las mesas están dispuestas en grupos de 4-6 alumnos, en cualquier momento se pueden colocar en otras posiciones, por ejemplo por parejas, para explicar algo a un compañero o podrán irse a la zona de trabajo en equipo, donde hay mesas para trabajar juntos y a la vez algo retirados del bullicio del aula, pero dentro de la misma. La idea de la "multitarea" ayuda claramente en la presencia, el aprendizaje y la participación de todos los alumnos en el aula, pues ellos pueden elegir aquella tarea para la que se sienten más capacitados.

Otros proyectos interesantes en esta búsqueda de diferentes formas de enseñar y afrontar el aprendizaje de competencias, son los que complementan las estrategias de trabajo más globales y participativas, como las que hemos señalado, con otros espacios que permiten a cada alumno desarrollar determinadas competencias, a la vez que potencian su libertad de elección. En esta línea se encuentran las denominadas "Tardes Diversas", en donde se ofrecen talleres de muy diversa índole (taller de las letras, tarde de las ciencias, juegos cooperativos, taller de emprendedores, manualidades, taller corporal, etc.). Los alumnos eligen dos según sus preferencias, para cursarlos dos tardes a la semana.

\section{d) Profesorado que confía y escucha con sinceridad lo que sus alumnos y alumnas tienen que decirle para mejorar su prácticas de enseñanza y evaluación}

Como sabemos (Ainscow et al., 2012; Echeita et al.; Susinos y Ceballos, 2012), contar con las voces de los estudiantes se ha mostrado como una herramienta de gran valor en los procesos de revisión y mejora de los centros educativos. Aunque, como señalan Susinos y Ceballos (2012), debemos avanzar hacia otros modelos más comprometidos con la participación que vayan más allá de la consulta y, en donde el protagonismo de los alumnos en el diseño y desarrollo de proyectos del centro sea mayor.

En el CFPP cada vez que termina un periodo de evaluación se les pasa un cuestionario a los alumnos preguntándoles acerca de muchos aspectos, entre otros, el profesorado. Esta información la comparte el tutor con los profesores del equipo educativo siempre con vistas a la mejora educativa. Además, tanto alumnos como familias realizan encuestas de satisfacción cada curso escolar donde reflejan su opi- 
nión. El resultado de dichas encuestas de alumnos es comunicado por el Director del Centro a cada uno de los profesores, con idea de reconocer y felicitar por lo logrado, así como ofrecer recursos en caso de que haya aspectos que mejorar.

\section{e) Apertura de los centros a su comunidad y preocupación por la participación de las familias en un marco de confianza mutua y responsabilidad compartida}

Los tres centros están de acuerdo en que es necesario buscar diferentes y variadas vías para que las familias se impliquen, participen de una forma activa y real en todos los ámbitos de la escuela (Simón, 2013), avanzando hacia modelos de co-gestión (Bolìvar, 2006). Pero también hay una postura común al plantearse que es necesario ir más allá de las "puertas" de sus centros, hay que conocer los recursos de la comunidad y ponerlos a disposición de la escuela, a la vez que la propia escuela se "pone al servicio" de su comunidad (Parrilla, Muñoz y Sierra, 2013). El desarrollo de las escuelas debe estar ligado a los esfuerzos de la comunidad en la que se insertan para enfrentarse a las inequidades experimentadas por los niños. Esto implica vincular el trabajo de las escuelas con los de otras agencias, organizaciones y grupos comunitarios relacionados con la promoción del bienestar económico y social de la zona (Ainscow et al., 2012), así como promover la colaboración entre escuelas, proponiendo un enfoque de reciprocidad entre las mismas (Ainscow et al. 2012; Parrilla et al., 2013).

En el CEPL se programan eventos a lo largo del curso escolar importantes en la vida de los niños y su entorno en la que parte fundamental es la colaboración y participación de las familias. Se organiza de forma conjunta con las familias, con objetos y comida que todos aportan a un Mercadillo Solidario una vez al año con la finalidad de recaudar dinero para las diferentes salidas de fin de curso. Se celebra también con "Ios mayores" de la ESO y de los Ciclos Formativos el Día de puertas abiertas, donde los padres y madres pueden entrar en las aulas como un "alumno" más y compartir un día con ellos. También se cuida el ámbito de la formación de padres y madres. Por la singularidad del centro, bilingüe, y al haber también padres/madres sordos, se fomenta la comunicación y convivencia entre ellos, y se ofrece Formación en LSE a todas las personas oyentes.

En el CFPP las reuniones con las familias del primer ciclo, tanto al principio como al final de curso, son interactivas. Es decir, se crean grupos cooperativos de trabajo entre los padres asistentes y se les intenta explicar de este modo- como hemos mencionado anteriormente-, cómo aprenden sus hijos e hijas. Además, tienen también la posibilidad de participar trimestralmente en la denominada "Escuela de Familias" sobre temáticas de interés relacionadas con sus hijos e hijas. Al pasar el tutor tantas horas con su grupo de alumnos (en $1^{\circ}$ ESO toda la jornada y en $2^{\circ}$ la mitad de la jornada) el conocimiento del alumnado es muy grande y el contacto con las familias constante. Cuando las familias con hijos o hijas considerados con n.e.e. sienten la necesidad de una ayuda específica, tienen a su disposición el recurso tanto del profesorado de apoyo del centro como el que presta el Centro de Atención a Familias (en colaboración con la Universidad Pontificia Comillas).

En el CCS la estrategia seguida es tratar promover la participación de las familias mediante la realización de talleres de padres, tertulias literarias, grupos interactivos, 
formación para familiares, en línea con las propuestas que se hacen desde el marco de las "Comunidades de Aprendizaje", tal y como hemos recogido con anterioridad. En este momento están dando un paso más tratando de proponer iniciativas de participación de las familias en la toma de decisiones y en la evaluación de procesos clave de la escuela (a través comisiones mixtas, con alumnos incluidos).

\section{f) Equipos directivos que contribuyen a crear las condiciones y el clima de rela- ciones necesario para hacer frente a los constantes desafíos del proceso hacia una educación más inclusiva}

Como señalan Ainscow y West (2008), es estratégico saber aprovechar el potencial y las experiencias existentes dentro de cada escuela. Para ello, como reconocen los centros, el papel del equipo directivo es decisivo, pues deben ser capaces de crear las condiciones y el clima de relaciones necesario para que todas estas actividades necesarias para el desarrollo profesional de los docentes y la mejora del centro puedan Ilevarse a cabo. Como señalan Murillo y Krichesky (2015), en todos los procesos de cambio escolar considerados eficaces siempre hay uno o una líder que es capaz de promover y sostener una cultura de aprendizaje y mejora del centro.

El caso del CEPL se asume un proceso continuo de cambio del centro, promovido y apoyado por el equipo directivo. Con la finalidad de mejorar la práctica docente y el clima general del centro se atiende a la formación de todos los profesionales partiendo de las necesidades propuestas de cada etapa educativa, así como el trabajo en equipo. Asimismo, este equipo facilita las decisiones compartidas y consensuadas en la Comisión de Coordinación Pedagógica en la que están representadas todas las etapas educativas a través de su coordinador, así como el Departamento de orientación. El coordinador recoge propuestas de su etapa y tienen autonomía en algunas decisiones. Además, el equipo directivo forma parte del equipo de profesorado ya que dan asignaturas en varias etapas lo que hace que conozcan de primera mano las dificultades y que el grupo de profesores les vea como "uno más".

Tanto en el caso del CFPP, como del CCS se reconoce que el ánimo, el impulso y el liderazgo del equipo directivo, sobre todo en los comienzos del proyecto, ha sido básico para su buen funcionamiento a lo largo de todos estos años a través de una gestión de los horarios del profesorado, los materiales, los espacios y los recursos en coherencia con los objetivos, la visión y los valores compartidos en el centro. En definitiva, se trata de que el proyecto sea un proyecto de Centro, más allá de las individualidades.

En el CFPP el equipo de profesores que forma parte del proyecto de Aulas Cooperativas Multitarea dedicó bastantes horas de formación sobre todo en aprendizaje cooperativo en los primeros años de la implantación del proyecto. A partir de ahí, desde la Jefatura de Estudios se proporcionó a todos los equipos de profesores una hora semanal para la coordinación de materias, ámbitos de aprendizaje, planificación semanal y para las reflexión sobre la propia acción. A final de curso, todos los años, la Coordinadora de etapa elabora, con el equipo educativo de $1^{\circ}$ y $2^{\circ}$, un DAFO para, partiendo de él, elaborar las propuestas de mejora para el curso siguiente.

Como resultado de todo lo anterior, en los centros se encuentra una actitud hacia la innovación como parte inherente a la acción educativa. Se asume que la educa- 
ción inclusiva implica entenderla como un proceso de continuo cambio (en la introducción hablábamos de transformación), que no tiene fin, pues no es una meta que podamos alcanzar como tal. La esencia de la inclusión está en el propio proceso de "estar en marcha", revisando continuamente las barreras que pueden generar, segregación, marginación o fracaso escolar e introduciendo cambios de forma planificada y rigurosa, lo que implica evaluar lo logrado.

En la Tabla 1 se presentan de forma resumida algunas de prácticas específicas (no todas) organizadas en torno a los diferentes niveles de la gramática escolar que conforman la vida de una escuela: sus culturas, sus políticas y sus prácticas. Además, para facilitar el contraste con las visiones más tradicionales, hemos contrapuesto cada acción Ilevada a cabo con aquella que han tratado de superar, de forma que nos permite tener una visión de conjunto de "ambos tipos de escuelas".

Tabla 1. Prácticas de las escuelas que configuran una "gramática inclusiva" en contraposición con una "gramática tradicional"

\begin{tabular}{|c|c|}
\hline GRAMÁTICA “TRADICIONAL” & GRAMÁTICA “INCLUSIVA” \\
\hline \multicolumn{2}{|c|}{ Cultura de centro } \\
\hline La diversidad como "problema". & $\begin{array}{l}\text { La diversidad como "oportunidad", como } \\
\text { riqueza. }\end{array}$ \\
\hline $\begin{array}{l}\text { Las "dificultades de aprendizaje" explicadas } \\
\text { fundamentalmente en términos de déficits o } \\
\text { condiciones personales o sociales del alum- } \\
\text { nado. }\end{array}$ & $\begin{array}{l}\text { Las "dificultades de aprendizaje" explicadas } \\
\text { fundamentalmente en términos de función } \\
\text { o interacción entre las condiciones persona- } \\
\text { les del alumnado y la respuesta educativa } \\
\text { ofertada }\end{array}$ \\
\hline $\begin{array}{l}\text { Expectativas deterministas ante la capaci- } \\
\text { dad de aprender del alumnado: expectativas } \\
\text { limitadas respecto a sus posibilidades de } \\
\text { aprendizaje. }\end{array}$ & $\begin{array}{l}\text { Expectativas transformadoras ante la capa- } \\
\text { cidad de aprender del alumnado: "Hurt y } \\
\text { Drummond, 2014) }\end{array}$ \\
\hline $\begin{array}{l}\text { Limitación de la participación de la comu- } \\
\text { nidad educativa. Concepción limitada de la } \\
\text { participación }\end{array}$ & $\begin{array}{l}\text { Concepción de participación basada en la } \\
\text { implicación de toda la comunidad educati- } \\
\text { va en todos los niveles del centro. }\end{array}$ \\
\hline $\begin{array}{l}\text { Los alumnos o familias se consideran ajenos } \\
\text { a las mejoras del centro. Ausencia de par- } \\
\text { ticipación real de la comunidad educativa. }\end{array}$ & $\begin{array}{l}\text { Las "voces" de alumnado, familia, profesio- } \\
\text { nales son una estrategia fundamental para la } \\
\text { revisión y mejora. }\end{array}$ \\
\hline \multicolumn{2}{|c|}{ Políticas de centro } \\
\hline $\begin{array}{l}\text { Profesorado que trabaja, mayoritariamente, } \\
\text { aislado, dentro y fuera del aula. }\end{array}$ & $\begin{array}{l}\text { Profesorado que trabaja en colaboración } \\
\text { con otros, dentro y fuera del aula. }\end{array}$ \\
\hline Un solo profesor en el aula. & $\begin{array}{l}\text { Dos o más profesores por aula, los profeso- } \\
\text { res de apoyo trabajan en el aula (apoyando } \\
\text { también a otros alumnos o alumnas), volun- } \\
\text { tarios como apoyo dentro del aula. }\end{array}$ \\
\hline $\begin{array}{l}\text { Una sola materia por profesor, sin coordina- } \\
\text { ción con otras }\end{array}$ & Materias integradas/ámbitos \\
\hline
\end{tabular}




\begin{tabular}{|l|l|}
\hline $\begin{array}{l}\text { La participación de las familias como con } \\
\text { los trastos viejos, "pocas y lejos" }\end{array}$ & $\begin{array}{l}\text { Amplia y consistente participación e impli- } \\
\text { cación de las familias en la vida del centro }\end{array}$ \\
\hline $\begin{array}{l}\text { Un “modelo experto y remedial" en el ámbi- } \\
\text { to de la orientación educativa }\end{array}$ & $\begin{array}{l}\text { Un “modelo colaborativo y educativo" en el } \\
\text { ámbito de la orientación educativa }\end{array}$ \\
\hline \multicolumn{2}{|c|}{ Prácticas de aula } \\
\hline $\begin{array}{l}\text { Alumnado pasivo, receptivo a las "explica- } \\
\text { ciones del profesor". Trabajo rutinario }\end{array}$ & $\begin{array}{l}\text { Alumnado activo, participativo, aprendien- } \\
\text { do del profesor y de sus compañeros o com- } \\
\text { pañeras. Trabajo por proyectos. }\end{array}$ \\
\hline $\begin{array}{l}\text { Estructura de interdependencia entre el alum- } \\
\text { nado de carácter individual o competitivo. }\end{array}$ & $\begin{array}{l}\text { Estructura de la interdependencia entre el } \\
\text { alumnado de carácter cooperativo. }\end{array}$ \\
\hline $\begin{array}{l}\text { Un solo sistema de comunicación } \\
\text { El libro de texto como fuente principal de } \\
\text { conocimiento }\end{array}$ & $\begin{array}{l}\text { Diversidad de sistemas de comunicación } \\
\text { cuando se necesitan (LSE) } \\
\text { Diversidad de recursos y fuentes de infor- } \\
\text { mación para enseñar y aprender }\end{array}$ \\
\hline $\begin{array}{l}\text { El aula como único espacio de aprendizaje } \\
\text { El contexto, el centro y el aula como espa- } \\
\text { cios potenciales de aprendizaje }\end{array}$ \\
\hline
\end{tabular}

LeCCIONES APRENDIDAS EN EL PROCESO haCIA CULTURAS, POLÍtICAS Y PRÁCTICAS MÁS INCLUSIVAS

A lo largo del "viaje" particular emprendido por cada escuela, en este proceso de cambio, mejora e innovación iniciado, hacia culturas, políticas y prácticas más inclusivas hemos podemos identificar colaborativamente lo que podríamos llamar "algunas lecciones aprendidas" durante este tiempo, que en cada caso están encarnadas en realidades y contextos singulares pero que, al mismo tiempo, son muy semejantes.

Entre ellas se encontrarían las siguientes:

- "El cambio es posible", a pesar de las múltiples barreras (estructurales, del sistema educativo, vinculadas a recursos, tradiciones, viejas concepciones entre el profesorado y otros agentes educativos, etc.).

- Los valores son un sustento central para manteneros en el empeño de cambiar.

- Para iniciar los cambios ha sido necesario "soñar una escuela distinta" al igual que tener expectativas positivas, de éxito.

- Pese al esfuerzo, dilemas, encuentros y desencuentros, "ha merecido y sigue mereciendo la pena", y que los efectos/resultados (en distintos planos educativos y tanto para el conjunto del alumnado como para el conjunto de la comunidad educativa) están siendo enriquecedores y moralmente reconfortantes.

- Ha sido imprescindible "romper" "cambiar" elementos relevantes de la gramática escolar existente previamente en el centro.

- Las emociones han jugado un papel muy relevante en todo el proceso y que, aunque ha habido y en ocasiones sigue habiendo cansancio, frustración, desaliento, etc., también ha habido y hay mucha esperanza, ilusión, implicación, motivación, perseverancia... 
- No puede ser un empeño de unos pocos "visionarios o caudillos", sino una empresa colaborativa, comunitaria, de ahí la necesidad de implicar fuertemente a las familias, entre otros agentes educativos.

- Sin el apoyo del equipo directivo estos procesos no son sostenibles y se quedan finalmente en experiencias aisladas sin continuidad.

- La posibilidad de un espacio para compartir y reflexionar entre centros, intercambiar experiencias, compartir saberes, añade un elemento más a la red de apoyos que nos permite avanzar.

- Hay muchas cosas que cambiar aún, no es fácil ni se debe caer en el engaño de un falo optimismo. Hay personas, prácticas y tradiciones muy resistentes al cambio y, como es de esperar, también hay cosas más fáciles en las etapas tempranas y otras más difíciles en las etapas superiores de la educación obligatoria.

- La educación inclusiva no es una "teoría o nueva moda" construida por "académicos" alejados de la realidad de los centros. La educación inclusiva se la estamos haciendo día a día en nuestros centros, en nuestra aula, perfectible, pero real.

Si bien hemos tratado de compartir algunas prácticas que, con mayor o menor, acierto se están poniendo en marcha en nuestros centros, en ningún caso hemos pretendido mostrarlas como un modelo a seguir. Tan solo hemos querido rescatar lo que, en nuestro caso, consideramos que merece la pena compartir con otros con la finalidad de mostrar diferentes formas de tratar de acotar la distancia entre los valores que asumimos y las prácticas que implementamos. A diferencia de quienes se empeñan en mostrar "buenas prácticas" que, sin embargo, luego no se pueden extrapolar a otros contextos y centros, nosotros simplemente hemos querido compartir nuestra realidad con la humilde esperanza de que, tal vez, tengan la capacidad de estimular la reflexión de que "otra gramática escolar", más inclusiva, es posible, para colaborar al desarrollo de una sociedad más justa e igualmente inclusiva.

Evidentemente, hay muchas cosas que mejorar, muchas barreras por superar vinculadas al deseo de maximizar con equidad la presencia, el aprendizaje y a la participación de alumnado, el profesorado, las familias y otros miembros de la comunidad en el devenir de la educación escolar que ofrecemos en nuestros centros. Ojalá que pronto podamos compartir con otros muchos este sueño que intentamos llevar a la práctica cada día del curso escolar.

\section{REFERENCIAS BIBLIOGRÁFICAS}

Ainscow, M., Dyson, A., Goldrick, S., y West, M. (2012). Making schools effective for all: rethinking the task. School Leadership \& Management, 32(3), 197-213.

Ainscow, M., y West, M. (2008). Improving urban schools. Leadership and collaboration. Maidenhead: Open University Press.

Booth, T. (2006). Manteniendo el futuro con vida; convirtiendo los valores de la inclusión en acciones. En M. A. Verdugo y F. B. Jordán de Urríes (Coords.), Rompiendo inercias. Claves para avanzar. VI Jornadas Científicas de Investigación sobre Personas con Discapacidad (pp. 211-217). Salamanca: Amarú. 
Booth, T., y Ainscow, M. (2011). Index for Inclusion. Developing learning and participation in schools ( $3^{\mathrm{a}} \mathrm{ed}$.). Bristol: CSIE.

Echeita, G., y Navarro, D. (2015). Educación inclusiva y desarrollo sostenible. Una llamada urgente a pensarlas juntas. Edetania, 46.

Echeita, G., Simón, C., López, M., y Urbina, C. (2013). Educación inclusiva. Sistemas de referencia, coordenadas y vórtices de un proceso dilemático. En M. A. Verdugo y R. L. Schalock (Eds.), Discapacidad e inclusión. Manual para la docencia (pp. 329-357). Salamanca: Amarú.

Echeita, G., Simón, C. Sandoval, M., y Monarca, H. (2013). Cómo fomentar las redes naturales de apoyo en el marco de una escuela inclusiva: propuestas prácticas. Sevilla: Eduforma.

Hart, S., y Drummond, M. J. (2014). Learning Without Limits: Constructing a Pedagogy Free from Determinist Beliefs about Ability. En L. Florian (Ed.), The SAGE Handbook of Special Education (2a ed.) (pp. 439-458). London: SAGE.

Flecha, R. (2009). Cambio, inclusión y calidad en las Comunidades de Aprendizaje. Cultura y Educación, 21(2), 157-169.

Foreman, P. (2011). Inclusion in action ( $3^{\mathrm{a}}$ ed.). Melbourne, Vic: Thompson.

Hernández, F. (2002). Los proyectos de trabajo. Mapa para navegantes en mares de incertidumbre. Cuadernos de Pedagogía, 310, 70-82.

INCLUD-ED Consortium (2011). Actuaciones de éxito en las escuelas europeas. Madrid: Ministerio de Educación IFIIE. Recuperado de: http://goo.gl/4GGA7a.

Kagan, S. y Kagan, M. (2009). Kagan cooperative learning. San Clemente, CA: Kagan Publishing.

Murillo, J., y Krichesky, G. J. (2015). Mejora de la Escuela: medio siglo de lecciones aprendidas. REICE. Revista Iberoamericana sobre Calidad, Eficacia y Cambio en Educación, 13(1), 69-102.

Norwich, B. (2008). Dilemmas of difference, inclusion and disability: international perspective placement. European Journal of Special Needs Education, 23(4), 287-304.

Sandoval, M., Simón, C., y Echeíta, G. (2012). Análisis y valoración crítica de las funciones del profesorado de apoyo desde la educación inclusiva. Revista de Educación, vol. extr., 117-137.

Simón, C. (2013). Las familias ante la inclusión educativa: de la incertidumbre a la implicación. En G. Echeita, C. Simón, M. Sandoval y H. Monarca (Eds.), Cómo fomentar las redes naturales de apoyo en el marco de una escuela inclusiva: propuestas prácticas (pp. 131-130). Sevilla: Eduforma.

Simón, C., y Echeíta, G. (2013). Comprender la educación inclusiva para intentar llevarla a la práctica. En H. Rodríguez y L. Torrego (Coords.), Educación inclusiva, equidad y derecho a la diferencia. Transformando la escuela (pp. 33-65). Madrid: Wolters Kluwer España.

Slee, R. (2012). La escuela extraordinaria. Exclusión, escolarización y educación inclusiva. Madrid: Morata.

Susinos, T., y Ceballos, N. (2012). Voz del alumnado y presencia participativa en la vida escolar. Apuntes para una cartografía de la voz del alumnado en la mejora educativa. Revista de Educación, 359, 24-44. 
Parrilla, M. A., Muñoz, M. A., y Sierra, S. (2013). Proyectos educativos con vocación comunitaria. Revista de Investigación en Educación, 3(11), 15-31.

Powell, J. (2013). Comparative and international perspective on Special Education. En L. Florian (Ed.), The SAGE Handbook of Special Education (2ª ed.) (pp. 335-349). London: SAGE.

Tyack, D., y Cuban, L. (2001). "Por qué persiste la gramática de la escolaridad", en En busca de la utopía. Un siglo de reformas de las escuelas públicas, México: Fondo de Cultura Económica.

UNESCO (2005). Guidelines for inclusion: Ensuring Access to Education for All. París: UNESCO. Recuperado de http://goo.gl/OjF0Ms.

Winter, E., y O'Raw, P. (2010). Literature review of the principles and practices relating to inclusive education for children with special educational needs. National Council for Special Education. Recuperado de http://goo.gl/ByTSbu. 